\title{
Anti-platelet and anti-inflammatory properties of an ethanol-water red grape pomace extract
}

\section{Abstract}

Previous reports support that wine contains a mixture of micro-constituents in a proper quality and quantity that possess cardioprotective effect partly through Platelet-Activating Factor (PAF) inhibition. Grape pomace (GP) is a source of wine-like microconstituents that may be a suitable alternative in food fortification. Limited data exist concerning their effects on thrombosis and inflammation. Therefore, the purpose of this study was to examine a grape pomace extract regarding its anti-platelet and anti-inflammatory properties. GP from four red grape varieties were extracted with 80\% ethanol (GP:solvent 1:5w/v). The extract's total phenolic compounds were evaluated and the phenolic profile was performed by Ultrahigh-Performance-Liquid-Chromatography coupled to Mass-Spectrometry and the determination of fatty acids profile was performed by Gas-Chromatography. The extract's anti-platelet properties were tested in healthy volunteers' platelet rich plasma by the light transmittance method, against three agonists: PAF, ADP and TRAP. The results expressed as $\mathrm{IC}_{50}$ values ( $\mu \mathrm{g}$ of extract that cause $50 \%$ inhibition of aggregation) and $\mathrm{EC}_{50}$ values (agonist concentration that causes $50 \%$ of the maximum aggregation) in the extract's presence and absence. Concerning the extract's anti-inflammatory properties, peripheral blood mononuclear cells from healthy volunteers were pre-incubated with different extract concentrations, which were tested for their effect on cell viability, for $1 \mathrm{~h}$ and then stimulated with LPS for $4 \mathrm{~h}$. Secretion of IL-1 $\beta$ and TNF-a was measured and normalized with the total cell protein. Phenolic compounds were calculated at $8.79 \pm 1.17 \mathrm{mg}$ gallic acid per $\mathrm{g}$ of GP. The most abundant ones were catechin, epicatechin and quercetin at $202.9 \pm 6.9,84.8 \pm 1.5$ and $83.7 \pm 3.5 \mu \mathrm{g}$ per g of GP respectively. Out of the 18 fatty acids detected, the most abundant ones were palmitic, oleic, linoleic, and linolenic acid at $28.7 \pm 0.1,11.4 \pm 0.01,32.5 \pm 0.07,12.7 \pm$ $0.005 \mathrm{~g}$ per $100 \mathrm{~g}$ of fat. The extract's $\mathrm{IC}_{50}$ was calculated at $162.1 \pm 66.9,181.2 \pm 82.3$ and $156.3 \pm 97.5 \mu \mathrm{g}$ against PAF, ADP and TRAP, respectively. The $\mathrm{EC}_{50}$ values in the presence of $150 \mu \mathrm{g}$ extract were increased (lower platelet aggregation sensitivity) approximately at $100 \%, 45 \%$ and $13 \%$ against PAF, ADP and TRAP respectively, compared to $\mathrm{EC}_{50}$ values in the absence of extract. The presence of 500 and $1000 \mu \mathrm{g} / \mathrm{mL}$ of extract reduced LPS-induced TNF-a secretion at approximately $38.2 \%(\mathrm{p}=0.04)$ and $6.0 \%(\mathrm{p}<0.000)$, respectively. Potent anti-platelet and anti-inflammatory properties are combined in a grape pomace extract. The use of its bioactive microconstituents is likely to lead to the production of functional foods with cardioprotective properties.

\section{Conflict of Interest}

There is no conflict of interest 